\title{
Análise espacial de parâmetros da fertilidade do solo em região de ecótono sob diferentes usos e manejos
}

\section{Spatial analysis of parameters of soil fertility in an ecotone under different uses and management}

\author{
Sabino Pereira da Silva Neto ${ }^{1 *}$; Antonio Clementino dos Santos ${ }^{2}$; Raimundo \\ Laerton de Lima Leite ${ }^{1}$; Valdinéia Patrícia Dim; Rossini Sôffa da Cruz ${ }^{1}$; Angélica \\ Pedrico $^{1}$; Durval Nolasco das Neves Neto ${ }^{1}$
}

\begin{abstract}
Resumo
Em regiões de ecótono as características químicas do solo apresentam uma ampla variação, resultante da interrelação entre os fatores de formação, cobertura vegetal e uso. O objetivo do presente estudo foi avaliar a variabilidade espacial de atributos químicos de Neossolo Quartzarênico Órtico típico na zona de transição entre o Bioma Cerrado e o Amazônico sob diferentes formas de uso e manejo (floresta, capoeira, solo desnudo, cultura agrícola, pastagem e cerradão) na região de Araguaína (TO). Previamente a implantação do experimento foram realizadas amostragens de solo em uma malha irregular com área de 26,08 ha, na profundidade de 0 a $20 \mathrm{~cm}$ para determinação dos teores de matéria orgânica (M.O.), $\mathrm{P}, \mathrm{K}^{+}, \mathrm{Ca}^{2+}, \mathrm{Mg}^{2+}$ e os valores de $\mathrm{pH}$, Soma de bases $(\mathrm{SB})$, capacidade de troca de cátions $\left(\mathrm{CTC}_{\mathrm{pH}}\right.$ $\left.{ }_{7.0}\right)$, capacidade de troca de cátions efetiva $\left(\mathrm{CTC}_{\mathrm{e}}\right)$, saturação por base $(\mathrm{V} \%)$ e saturação por alumínio $(\mathrm{m} \%)$. Os dados obtidos de cada um dos atributos químicos do solo foram submetidos à analises de estatística descritiva, à comparação de média pelo teste de Tukey ao nível de 5\% de significância, ao estudo geoestatístico e interpolação por krigagem. Os alcances de dependência espacial dos atributos químicos M.O. $\mathrm{Al}^{3+}, \mathrm{K}^{+}, \mathrm{Mg}^{2+}, \mathrm{SB}$ e $\mathrm{CTC}_{\mathrm{e}}$ do solo foram maiores que os demais ( $\mathrm{pH}, \mathrm{P}, \mathrm{Ca}^{2+}, \mathrm{CTC}_{\mathrm{pH}}$ 7, $\mathrm{V} \%$ e $\mathrm{m} \%$ ), indicando que esses apresentam maior descontinuidade na distribuição espacial em Neossolo Quartzarênico Órtico típico sob três formas de uso agrícola e de três coberturas vegetais de zona de transição Cerrado-Amazônia. As estratégias de manejo de correção e fertilização mineral em região de ecótono podem ser montadas em função das representações dos estudos geoestatísticos e de interpolações por krigagem nas zonas mais homogêneas.
\end{abstract}

Palavras-chave: Geoestatística. Krigagem. Manejo do solo. Variabilidade especial. Variograma.

\begin{abstract}
In regions of ecotone soil chemical properties show a wide variation, resulting from the interplay between the factors of formation, vegetation cover and use. The aim of this study was to evaluate the spatial variability of chemical attributes of a Entisol transition zone between the Savannah and Amazonian biome in different forms of land use and management (forest, scrub, bare soil, crop, pasture and savanna) in the region of Araguaína city (TO). Samples were ground in an irregular mesh in an area of 26.08 ha, the depth of 0 to $20 \mathrm{~cm}$ to determine the levels of organic matter, $\mathrm{P}, \mathrm{K}^{+}, \mathrm{Ca}^{2+}, \mathrm{Mg}^{2+}$ and $\mathrm{pH}$, sum of bases, $\mathrm{CEC}_{\mathrm{pH}}, \mathrm{CEC}_{\mathrm{e}}, \mathrm{V} \%, \mathrm{~m} \%$. The soil chemical properties were analyzed using descriptive statistics, comparison of average by Tukey test at $5 \%$ significance to the study and geostatistical kriging.
\end{abstract}

\footnotetext{
1 Doutorando em Ciência Animal. Universidade Federal do Tocantins. Rod. BR 153, Km 112, Caixa Postal 132. E-mail: sabinozootec@yahoo.com.br; laerton.leite@bol.com.br; valpatdim@hotmail.com; rossinizoot@hotmail.com; angelpedrico@ hotmail.com; durval.nolasco@gmail.com

2 Prof. da Universidade Federal do Tocantins. Rod. BR 153, Km 112, Caixa Postal 132. E-mail: clementino@uft.edu.br

* Autor para correspondência
} 
The ranges of spatial dependence of the chemical organic matter, $\mathrm{Al}^{3+}, \mathrm{K}^{+}, \mathrm{Mg}^{2+}$, and $\mathrm{SB}, \mathrm{CEC}$ soil were higher than the others $\left(\mathrm{pH}, \mathrm{P}, \mathrm{Ca}^{2+}, \mathrm{CEC}_{\mathrm{pH}}, \mathrm{V} \%, \mathrm{~m} \%\right)$, indicating that these are more discontinuity in the spatial distribution in Entisol three forms of agricultural use and vegetation cover three of the transition Savannah-Amazon. Thus, management strategies of correction and mineral fertilizers in an ecotone can be fitted according to the representations of studies and geostatistical kriging interpolation in homogeneous areas.

Key words: Geostatistics. Kriging, soil management. Spatial variability. Variogram.

\section{Introdução}

As propriedades químicas e biológicas do solo são invariavelmente alteradas após a retirada da cobertura vegetal original para o uso agrícola, as quais são dependentes das condições edafoclimáticas, do tipo de cultura e das práticas culturais adotadas. Nesse sentido, a interrelação destas condições estabelece uma nova condição de equilíbrio no sistema solo (MARCHIORI JÚNIOR; MELO, 2000).

O uso do solo, com o passar do tempo, conduz a aumento na heterogeneidade dos atributos físicos, químicos e biológicos, por meio dos processos de desmatamento, preparo do solo, rotações de cultura e localização de aplicação de fertilizantes (SANTOS; SALCEDO, CANDEIAS, 2002; CAVALCANTE et al., 2007). Por isso, tanto a variabilidade espacial quanto a temporal dos atributos do solo devem ser incorporadas aos procedimentos e tecnologias aplicados à agricultura (LI et al., 2002). Vieira (2000) relata que a variabilidade espacial das propriedades do solo vem sendo uma das preocupações de pesquisadores, praticamente, desde o início do século.

O conhecimento da variabilidade espacial dos atributos da fertilidade do solo pode contribuir na definição de melhores estratégias para o manejo sustentável do solo (SCHAFFRATH et al., 2008). No entanto, torna-se necessário o uso de ferramentas de geoestatística, pois a estatística clássica não permite observar a presença de dependência espacial, uma vez que ela não leva em consideração a distância na qual as amostras foram coletadas no campo.

A geoestatística vem sendo constantemente aplicada em estudos para verificar a distribuição espacial das propriedades químicas do solo. Essa aplicação está baseada na possibilidade de se estudar o comportamento da variabilidade espacial, permitindo ter o conhecimento detalhado dos atributos da fertilidade do solo em diferentes locais de certa zona de manejo, podendo assim, aperfeiçoar a aplicação de corretivos e fertilizantes. A caracterização da variabilidade espacial dos atributos do solo é essencial para alcançar a melhor compreensão das complexas relações entre as propriedades do solo e os fatores ambientais (GOOVAERTS, 1998).

As características químicas do solo podem variar de acordo com o seu uso, conforme constatado por Su et al. (2004). Assim, na zona de transição entre o Bioma Cerrado e o Amazônico, o manejo da cultura e do solo podem influenciar na variabilidade dos atributos químicos do solo de mesma classe taxonômica. O comportamento diferenciado dos atributos dos solos em diferentes segmentos da paisagem pode ser entendido pela caracterização da variabilidade espacial dos solos por meio de técnicas de geoestatística, identificando locais específicos na paisagem (MONTANARI et al., 2008).

As contribuições e incrementos significativos na produtividade e na qualidade agrícola a partir da abordagem geoestatística são pouco testadas e pesquisadas em condições de solos na zona de transição Cerrado-Amazônia. Diante disso, o objetivo deste trabalho foi estudar a variabilidade espacial das propriedades químicas em Neossolo Quartzarênico Órtico típico sob diferentes classes de uso em região de ecótono. 


\section{Material e Métodos}

A área de estudo está situada entre as latitudes $7^{\circ} 5^{\prime}$ $50^{\prime}$ 'S e $7^{\circ} 5^{\prime} 33^{\prime}$ 'S e longitudes $48^{\circ} 12^{\prime} 31^{\prime \prime} \mathrm{W}$ e $48^{\circ} 11^{\prime}$ 59" W, na fazenda da Escola de Medicina Veterinária e Zootecnia da Universidade Federal do Tocantins (EMVZ-UFT), Campus de Araguaína. De acordo com classificação climática proposta por Köppen (1948) a região apresenta clima tipo Aw (quente úmido), com temperatura média de $28^{\circ} \mathrm{C}$ e precipitação pluviométrica média de $1800 \mathrm{~mm}$ anuais. O solo do local é classificado como Neossolo Quartzarênico Órtico típico (EMBRAPA, 2006).

As amostras do solo foram retiradas na profundidade de 0-20 cm, com sonda de amostragem em 90 pontos georeferênciados em malha irregular de uma área total de 26,08 ha, ocupada por seis classes de uso do solo (Tabela 1). Assim sendo, em cada tipo de uso do solo foram determinados 15 pontos de coleta (Figura 1).

Tabela 1. Classes de uso do solo da área de estudo.

\begin{tabular}{lll}
\hline Classe de uso do solo & \multicolumn{2}{c}{ Área } \\
\cline { 2 - 3 } & ha & \% \\
\hline Floresta $^{1}$ & 4,46 & 17,10 \\
Capoeira $^{2}$ & 1,67 & 6,40 \\
Solo nu & 3,84 & 14,72 \\
Cultura agrícola & 3,00 & 11,50 \\
Pastagem $^{4}$ & 6,39 & 24,50 \\
Cerradão & 6,72 & 25,77 \\
TOTAL & 26,08 & 100 \\
\hline
\end{tabular}

${ }^{(1)}$ : floresta amazônica; ${ }^{(2)}$ : regeneração da vegetação natural, ${ }^{(3)}$ : cultivo de milheto, ${ }^{(4)}$ : pastagem de Brachiaria brizantha cv. Marandu.

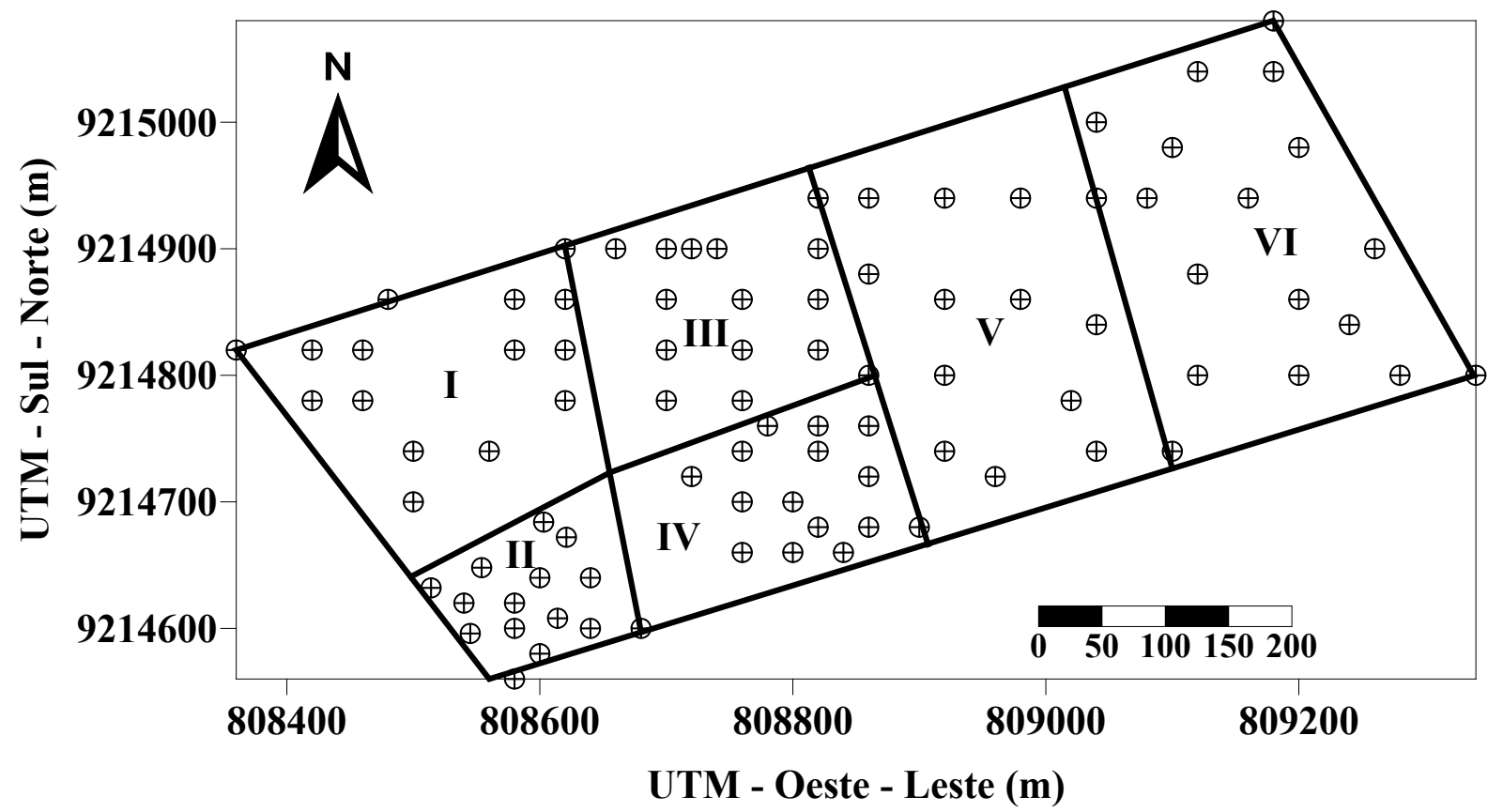

Figura 1. Local de coleta do solo nas classes de uso: Floresta (I), Capoeira (II), Solo nu (III), Cultura agrícola (IV), Pastagem (V) e Cerradão (VI). 
Após as coletas todas as amostras de solo, foram secas ao ar e peneiradas em peneira de malha de 2,0 mm e então obtidas amostras de terra fina seca ao ar (TFSA) para posteriores análises químicas no laboratório de solos da UFT. Nessas amostras de solo, foram determinados o valor de $\mathrm{pH}$ em $\mathrm{CaCl}_{2}$, os teores de $\mathrm{P}$ e $\mathrm{K}^{+}$extraíveis em Mehlich-1, os teores de $\mathrm{Ca}^{2+}, \mathrm{Mg}^{2+}$ e $\mathrm{Al}^{3+}$ trocáveis extraídos em $\mathrm{KCl} 1 \mathrm{~mol} \mathrm{~L}^{-1}$ e a matéria orgânica de acordo com as recomendações da Embrapa (1999). Com os valores obtidos nas análises do solo, calculouse a capacidade de trocas de cátions (CTC) a $\mathrm{pH}$ 7,0, a CTC efetiva, soma de bases trocáveis (SB), a porcentagem de saturação por alumínio $(\mathrm{m} \%)$ e percentagem de saturação por bases $(\mathrm{V} \%)$ (EMBRAPA, 1999).
Para cada atributo químico do solo estudado realizou-se análise estatística descritiva e a comparação de média pelo teste de Tukey ao nível de 5\% de significância, com auxilio do software Assistat versão 7.5 beta (SILVA, 2008). Na estatística descritiva calculou-se a média, a mediana, a variância, a assimetria, a curtose, o coeficiente de variação e o tipo de distribuição. Utilizou-se o teste de Kolmogorov-Smirnov (KS) a 5\% de probabilidade para testar a hipótese de normalidade.

O padrão de dependência espacial foi caracterizado por meio de análise geoestatística (VIEIRA, 2000) onde foi calculada a semivariância, utilizando o software GS+ (ROBERTSON, 1998), por meio da equação abaixo:

$$
\gamma(h)=\frac{1}{2 N(h)} \sum_{i=1}^{N(h)}\left[Z\left(x_{i}\right)-Z\left(x_{i}+h\right)\right]^{2}
$$

sendo $\mathrm{N}(\mathrm{h})$ o número de pares de valores experimentais medidos em [Z(xi), Z(xi $+\mathrm{h})]$, separados pelo vetor $h$. No presente estudo, os valores de $\mathrm{Z}$ foram os atributos químicos do solo estudado, enquanto os valores de xi e xi $+\mathrm{h}$ foram definidos de acordo com a localização geográfica das amostras realizadas no campo. O ajuste dos semivariogramas possibilitou definir os valores do efeito pepita $\left(\mathrm{C}_{0}\right)$, do alcance $(\mathrm{A})$ e do patamar $(\mathrm{C}$ $\left.+\mathrm{C}_{0}\right)$.

A seleção do modelo ajustado dos semivariogramas foi realizada com base na menor soma do quadrado dos resíduos (SQR), no maior coeficiente de determinação $\left(\mathrm{R}^{2}\right)$ e maior grau de dependência espacial (GDE). Conforme Robertson (1998) a proporção dada pela equação:

$$
G D E=\left[C /\left(C+C_{0}\right)\right] 100
$$

permite classificar o GDE em: dependência espacial fraca em GDE $\leq 25 \%$, dependência espacial moderada quando $25 \%<\mathrm{GDE} \leq 75 \%$ e dependência espacial forte em GDE $>75 \%$.

A interpolação dos valores foi realizada pelo método geoestatístico de Krigagem, de modo a definir o padrão espacial das variáveis estudadas, o que permitiu a elaboração dos mapas de isolinhas, utilizando-se o software Surfer 8.0 (GOLDEN SOFTWARE, 2002).

\section{Resultados e Discussão}

Os resultados da análise descritiva dos atributos químicos do solo são apresentados na Tabela 2. A aplicação do teste de normalidade de KolmogorovSmirnov ao conjunto de dados revela que apenas as variáveis M.O., $\mathrm{CTC}_{\mathrm{pH} 7}$ e $\mathrm{CTC}_{\mathrm{e}}$ apresentam distribuição normal, os demais parâmetros da fertilidade do solo da área estuda, não possuem distribuição normal. Estudando atributos químicos do solo Corá et al. (2004) obtiveram resultado bem semelhante já que somente a $\mathrm{CTC}_{\mathrm{pH} 7}$ e os teores de $\mathrm{K}^{+}$apresentavam distribuição normal. Já Oliveira 
et al. (1999), estudando a variabilidade espacial de atributos químicos, observaram distribuição normal somente na CTC efetiva de onze atributos químicos de solo salino-sódico. Para a área em estudo a falta de normalidade dos dados provavelmente ocorreu devido a aplicação não-uniforme de adubos ou mesmo pela diferentes formas de uso e manejo. Ocorrendo assim, no campo, locais com menores teores de nutrientes e outros com maiores. Por outro lado, o estudo geoestatístico de um conjunto dados não exige que esses tenham distribuição normal (CORÁ; BERALDO, 2006). O mais importante que a normalidade na distribuição dos dados, é a ocorrência ou não do chamado efeito proporcional, em que a média e a variabilidade dos dados são constantes na área de estudo. Esse evento não ocorreu neste estudo, uma vez que os semivariogramas apresentam patamares bem definidos e a distribuição não apresentou caudas muito alongadas, o que poderia comprometer as estimativas da krigagem, as quais são baseadas nos valores médios (ISAAKS; SRIVASTAVA, 1989).

Tabela 2. Estatística descritiva dos atributos da fertilidade do Neossolo Quartzarênico Órtico típico.

\begin{tabular}{|c|c|c|c|c|c|c|c|}
\hline \multirow[t]{2}{*}{ Variável } & \multirow[t]{2}{*}{ Média } & \multirow[t]{2}{*}{ Mediana } & \multirow[t]{2}{*}{ Variância } & \multicolumn{3}{|c|}{ Coeficiente } & \multirow[t]{2}{*}{$\mathbf{K S}^{\mathbf{1}}$} \\
\hline & & & & Variação & Assimetria & Curtose & \\
\hline M.O. $\left(\mathrm{g} \mathrm{dm}^{-3}\right)$ & 10,23 & 10,09 & 10,44 & 31,60 & 0,04 & $-0,04$ & $0,08 *$ \\
\hline $\mathrm{pH}\left(\mathrm{CaCl}_{2}\right)$ & 5,24 & 4,96 & 0,34 & 11,22 & 0,84 & $-0,31$ & $0,22 * *$ \\
\hline $\mathrm{Al}^{3+}\left(\mathrm{cmol}_{\mathrm{c}} \mathrm{dm}^{-3}\right)$ & 0,48 & 0,42 & 0,15 & 80,42 & 0,64 & $-0,46$ & $0,15 * *$ \\
\hline $\mathrm{P}\left(\mathrm{mg} \mathrm{dm}^{-3}\right)$ & 0,66 & 0,60 & 0,02 & 23,88 & 1,38 & 2,26 & $0,15^{* *}$ \\
\hline $\mathrm{K}+\left(\mathrm{cmol}_{\mathrm{c}} \mathrm{dm}^{-3}\right)$ & 0,002 & 0,0019 & 0,00 & 36,07 & 1,02 & 0,21 & $0,46^{* *}$ \\
\hline $\mathrm{Ca}^{2+}\left(\mathrm{cmol}_{\mathrm{c}} \mathrm{dm}^{-3}\right)$ & 0,54 & 0,46 & 0,21 & 84,56 & 0,50 & $-0,91$ & $0,21 * *$ \\
\hline $\mathrm{Mg}^{2+}\left(\mathrm{cmol}_{\mathrm{c}} \mathrm{dm}^{-3}\right)$ & 0,54 & 0,54 & 0,06 & 46,88 & 0,30 & $-0,85$ & $0,11^{* *}$ \\
\hline $\mathrm{SB}\left(\mathrm{cmol}_{\mathrm{c}} \mathrm{dm}^{-3}\right)$ & 1,08 & 0,87 & 0,44 & 62,32 & 0,50 & $-0,86$ & $0,16^{* *}$ \\
\hline $\mathrm{CTC}_{\mathrm{pH} 7}\left(\mathrm{cmol}_{\mathrm{c}} \mathrm{dm}^{-3}\right)$ & 6,60 & 6,32 & 3,48 & 28,28 & 0,34 & 0,53 & $0,07 *$ \\
\hline $\mathrm{CTC}_{\mathrm{e}}\left(\mathrm{cmol}_{\mathrm{c}} \mathrm{dm}^{-3}\right)$ & 1,55 & 1,54 & 0,19 & 28,22 & 0,63 & 0,20 & $0,07^{*}$ \\
\hline $\mathrm{V}(\%)$ & 16,28 & 15,17 & 93,82 & 59,50 & 1,05 & 1,94 & $0,10^{* *}$ \\
\hline m (\%) & 35,78 & 34,53 & 787,67 & 78,43 & 0,09 & $-1,73$ & $0,19 * *$ \\
\hline
\end{tabular}

${ }^{(1)} \mathrm{KS}$ : teste de normalidade de Kolmogorov Smirnov, $\left({ }^{*}\right)$ : significativo a $5 \%,(* *)$ : não-significativo a $5 \%$. M.O: matéria orgânica; $\mathrm{SB}$ : soma de base; $\mathrm{CTC}_{\mathrm{pH} 7}$ : capacidade de troca de cátions a $\mathrm{pH} 7 ; \mathrm{CTC}_{\mathrm{e}}$ : capacidade de troca de cátions efetiva; V: saturação por base; m: saturação por alumínio.

Os valores dos coeficientes de assimetria e curtose demonstram que os atributos químicos do solo têm distribuições assimétricas. Entretanto, a maioria das variáveis tem valores baixos, desses coeficientes, e bem próximos à zero, a não ser para os atributos $\mathrm{P}, \mathrm{K}^{+}$e saturação por bases $(\mathrm{V} \%)$ com valores de coeficiente de assimetria positivos e altos, ou seja, com concentração dos valores acima do valor médio dessas variáveis. Apesar disto, os valores da média e mediana de todos os atributos químicos estudados estão bem próximos, indicando que o conjunto de dados não apresentam assimetria acentuada. Little e Hills (1978) verificaram que quando os valores da média e mediana são semelhantes, os dados apresentam ou se aproximam da distribuição normal. Podendo isso, ser indicativo de que as medidas de tendência central não são dominadas por valores atípicos na distribuição (CAMBARDELLA et al., 1994), evidenciando que os atributos envolvidos no estudo estão se aproximando de uma distribuição normal indicativo de que os dados estão adequados para uso da geoestatística.

Os valores de coeficiente de variação $(\mathrm{CV})$ indicam que a maioria dos dados apresentam elevado 
grau de heterogeneidade, sendo o $\mathrm{pH}$ a única variável que teve CV médio (11,22\%) (GOMES,1984). Resultados similares foram encontrados por Carvalho, Silveira e Vieira (2002), Silva et al. (2003) e Souza et al. (2004). O alto valor do CV para os atributos químicos do solo é justificável por se tratar de estudo de áreas sob distintas formas de uso e de manejo de adubação, que resulta em aplicações de adubos e de corretivos de forma nãouniforme, o mesmo ocorre com a decomposição de restos culturais e da serapilheira dos estratos arbóreos, provocando assim alta variabilidade nos atributos do solo. Essa circunstância é comprovada quando se realiza a comparação entre as médias das variáveis das diferentes classes de uso e manejo do solo pelo teste de Tukey (Tabela 3). Perante isto, são observadas diferenças significativas $(p<0,05)$ entre a maioria dos atributos químicos nas distintas localidades do estudo.

Tabela 3. Características químicas nas classes de uso do Neossolo Quartzarênico Órtico típico.

\begin{tabular}{|c|c|c|c|c|c|c|c|}
\hline \multirow[t]{2}{*}{ Variável } & \multicolumn{6}{|c|}{ Classe de uso do solo } & \multirow[b]{2}{*}{$\mathrm{CV}(\%)$} \\
\hline & Floresta & Capoeira & Solo nu & Cultura Agrícola & Pastagem & Cerradão & \\
\hline M.O. $\left(\mathrm{g} \mathrm{dm}^{-3}\right)$ & $12,34 \mathrm{a}$ & $11,08 \mathrm{ab}$ & $9,27 \mathrm{bc}$ & $7,35 \mathrm{c}$ & 9,86 abc & $11,45 \mathrm{ab}$ & 28,01 \\
\hline $\mathrm{pH}\left(\mathrm{CaCl}_{2}\right)$ & $4,68 \mathrm{~b}$ & $4,84 \mathrm{~b}$ & $5,80 \mathrm{a}$ & $5,77 \mathrm{a}$ & $5,50 \mathrm{a}$ & $4,83 \mathrm{~b}$ & 6,97 \\
\hline $\mathrm{Al}^{3+}\left(\mathrm{cmol}_{\mathrm{c}} \mathrm{dm}^{-3}\right)$ & $0,78 \mathrm{ab}$ & $0,63 \mathrm{~b}$ & $0,12 \mathrm{c}$ & $0,16 \mathrm{c}$ & $0,25 \mathrm{c}$ & $0,96 \mathrm{a}$ & 46,29 \\
\hline $\mathrm{P}\left(\mathrm{mg} \mathrm{dm}^{-3}\right)$ & $0,56 \mathrm{~b}$ & $0,54 \mathrm{~b}$ & $0,75 \mathrm{a}$ & $0,82 \mathrm{a}$ & $0,73 \mathrm{a}$ & $0,57 \mathrm{~b}$ & 18,02 \\
\hline $\mathrm{K}^{+}\left(\mathrm{mg} \mathrm{dm}^{-3}\right)$ & $1,08 \mathrm{a}$ & $0,98 \mathrm{a}$ & $0,77 \mathrm{a}$ & $0,77 \mathrm{a}$ & $0,93 \mathrm{a}$ & $1,08 \mathrm{a}$ & 34,34 \\
\hline $\mathrm{Ca}^{2+}\left(\mathrm{cmol}_{\mathrm{c}} \mathrm{dm}^{-3}\right)$ & $0,26 \mathrm{~b}$ & $0,14 \mathrm{~b}$ & $0,89 \mathrm{a}$ & $0,90 \mathrm{a}$ & $0,86 \mathrm{a}$ & $0,21 \mathrm{~b}$ & 57,84 \\
\hline $\mathrm{Mg}^{2+}\left(\mathrm{cmol}_{\mathrm{c}} \mathrm{dm}^{-3}\right)$ & $0,33 \mathrm{~b}$ & $0,42 \mathrm{~b}$ & $0,71 \mathrm{a}$ & $0,72 \mathrm{a}$ & $0,68 \mathrm{a}$ & $0,36 \mathrm{~b}$ & 35,62 \\
\hline $\mathrm{SB}\left(\mathrm{cmolc} \mathrm{dm}^{-3}\right)$ & $0,43 \mathrm{~b}$ & $0,57 \mathrm{~b}$ & $1,62 \mathrm{a}$ & $1,63 \mathrm{a}$ & $1,67 \mathrm{a}$ & $0,47 \mathrm{~b}$ & 31,36 \\
\hline $\mathrm{CTC}_{\mathrm{pH} 7}\left(\mathrm{cmol}_{\mathrm{c}} \mathrm{dm}^{-3}\right)$ & $6,12 c$ & $4,5 \mathrm{~d}$ & $7,62 \mathrm{ab}$ & $6,13 \mathrm{c}$ & $8,75 \mathrm{a}$ & $6,43 \mathrm{bc}$ & 20,45 \\
\hline $\mathrm{CTC}_{\mathrm{e}}\left(\mathrm{cmol}_{\mathrm{c}} \mathrm{dm}^{-3}\right)$ & $1,21 \mathrm{c}$ & $1,20 \mathrm{c}$ & $1,75 \mathrm{ab}$ & $1,79 \mathrm{ab}$ & $1,93 \mathrm{a}$ & $1,43 \mathrm{bc}$ & 21,93 \\
\hline $\mathrm{V} \%$ & $7,24 \mathrm{~d}$ & $13,06 \mathrm{~cd}$ & $21,84 \mathrm{ab}$ & $28,12 \mathrm{a}$ & $19,47 \mathrm{bc}$ & $7,96 \mathrm{~d}$ & 37,97 \\
\hline $\mathrm{m} \%$ & $64,81 \mathrm{a}$ & $52,45 \mathrm{~b}$ & $7,17 \mathrm{c}$ & $10,91 \mathrm{c}$ & $13,64 \mathrm{c}$ & $65,71 \mathrm{a}$ & 31,90 \\
\hline
\end{tabular}

Médias seguidas de mesma letra na linha não diferem entre si ao nível de probabilidade de $5 \%$ pelo teste de Tukey. M.O: matéria orgânica; SB: soma de base; $\mathrm{CTC}_{\mathrm{pH}}$ : capacidade de troca de cátions a $\mathrm{pH} 7 ; \mathrm{CTC}_{\mathrm{e}}$ : capacidade de troca de cátions efetiva; V: saturação por base; m: saturação por alumínio; CV: coeficiente de variação.

De acordo com a CFSMG (1999) os valores médios, dos atributos químicos do solo na camada de 0-20 cm (Tabela 3), apresentam classe de fertilidade média para $\mathrm{pH}, \mathrm{Mg}^{2+}$ e $\mathrm{CTC}_{\mathrm{pH}}$; baixa para M.O., $\mathrm{Ca}^{2+}$, $\mathrm{SB}$ e $\mathrm{CTC}_{\mathrm{e}}$; muito baixa para $\mathrm{P}, \mathrm{K}^{+}$, $\mathrm{V}(\%)$ e alta de $\mathrm{m}(\%)$ ou seja com características da fertilidade do solo restritivas para o crescimento de plantas, uma vez que o complexo de troca do solo está ocupado pelos íons $\mathrm{H}^{+}$e $\mathrm{Al}^{3+}$. Os sistemas de Cultivo agrícola, Pastagem e Solo nu apresentam valores mais altos das propriedades químicos do solo (pH, $\mathrm{P}, \mathrm{Ca}^{+2}, \mathrm{Mg}^{+2}, \mathrm{SB}, \mathrm{CTC}_{\mathrm{pH}}, \mathrm{CTC}_{\mathrm{e}}, \mathrm{V} \%$ ) (Tabela 3) quando em comparação a Floresta, Capoeira e Cerradão, pois esta situação era esperada, visto que nessas áreas foram realizadas adubações e correções sistemáticas do solo.

Os elevados CV apresentados na Tabela 2 para as variáveis químicas do solo e os demonstrados no estudo de comparação de médias da Tabela 3 indicam que mesmo em estudos usando a estatística clássica que consideram as diferentes classes de uso e manejo do solo de forma isolada, podem ocorrer problemas devido ao uso de valores médios com alta variabilidade para a utilização em tomada de decisões, quanto ao manejo químico do solo tal como fertilização e correção. Desse modo, essa condição pode levar à aplicação de doses sub ou superestimadas e/ou até mesmo adequadas para 
locais específicos.

Para cada atributo estudado, ajustaram-se os modelos dos semivariogramas considerando os valores do coeficiente de determinação $\left(\mathrm{R}^{2}\right)$ e soma do quadrado dos resíduos (SQR) (Tabela 4). Robertson (1998) constatou que a SQR é um parâmetro mais robusto do que o $\mathrm{R}^{2}$ e propicia uma medida exata a qual o modelo se ajusta melhor aos dados. O programa $\mathrm{GS}^{+}$utiliza a combinação de parâmetros do semivariograma para minimizar a SQR para cada modelo.

Tabela 4. Modelos e parâmetros estimados dos semivariogramas ajustados aos valores dos atributos do Neossolo Quartzarênico Órtico típico.

\begin{tabular}{|c|c|c|c|c|c|c|c|c|}
\hline \multirow[t]{2}{*}{ Atributo } & \multicolumn{7}{|c|}{ Parâmetro } & \multirow[b]{2}{*}{$\mathrm{SQR}^{5}$} \\
\hline & Modelo & $\mathrm{C}_{0}{ }^{1}$ & $\mathrm{C}_{0}+\mathrm{C}^{2}$ & $A^{3}(m)$ & $\operatorname{GDE}^{4}(\%)$ & Classe & $\mathbf{R}^{2 *}$ & \\
\hline M.O. & Esférico & 6,73 & 17,24 & 1002 & 61 & Moderada & 0,95 & 2,32 \\
\hline pH & Esférico & 0,13 & 0,52 & 527 & 75 & Moderada & 0,96 & $5,14^{-3}$ \\
\hline $\mathrm{Al}^{3+}$ & Esférico & 0,003 & 0,48 & 1195 & 99 & Forte & 0,97 & $1,91^{-3}$ \\
\hline $\mathrm{P}$ & Esférico & 0,012 & 0,048 & 449 & 65 & Moderada & 0,99 & $5,18^{-6}$ \\
\hline $\mathrm{K}^{+}$ & Esférico & 0,063 & 0,17 & 1006 & 63 & Moderada & 0,86 & $7,29^{-4}$ \\
\hline $\mathrm{Ca}^{2+}$ & Esférico & 0,039 & 0,32 & 495 & 88 & Forte & 0,98 & $1,49^{-3}$ \\
\hline $\mathrm{Mg}^{2+}$ & Exponencial & 0,03 & 0,19 & 1071 & 84 & Forte & 0,90 & $3,22^{-4}$ \\
\hline SB & Esférico & 0,045 & 1,24 & 1060 & 96 & Forte & 0,99 & $5,38^{-3}$ \\
\hline $\mathrm{CTC}_{\mathrm{pH} 7}$ & Exponencial & 1,03 & 6,07 & 331 & 83 & Forte & 0,97 & 0,332 \\
\hline $\mathrm{CTC}_{\mathrm{e}}$ & Esférico & 0,09 & 0,38 & 1065 & 76 & Forte & 0,97 & $9,81^{-4}$ \\
\hline $\mathrm{V} \%$ & Exponencial & 39,30 & 267,90 & 827 & 85 & Forte & 0,95 & 422 \\
\hline $\mathrm{m} \%$ & Esférico & 1,00 & 2112 & 932 & 100 & Forte & 0,99 & 33045 \\
\hline
\end{tabular}

${ }^{(1)} \mathrm{C}_{0}$ : efeito pepita; ${ }^{(2)} \mathrm{C}_{0}+\mathrm{C}$ : patamar; ${ }^{(3)} \mathrm{A}$ : alcance; ${ }^{(4)} \mathrm{GDE}$ : grau de dependência espacial; ${ }^{(5)} \mathrm{SQR}$ : Soma do quadrado dos resíduos. $\left({ }^{*}\right) \mathrm{R}^{2}$ : coeficiente de determinação. M.O: matéria orgânica; SB: soma de base; $\mathrm{CTC}_{\mathrm{pH}}$ : capacidade de troca de cátions a pH 7; $\mathrm{CTC}_{\mathrm{e}}$ : capacidade de troca de cátions efetiva; $\mathrm{V}$ : saturação por base; m: saturação por alumínio.

Todos os atributos da fertilidade do solo analisados apresentaram dependência espacial na profundidade estudada $(0-20 \mathrm{~cm})$. Os atributos químicos M.O., pH, $\mathrm{Al}^{+}, \mathrm{P}^{+} \mathrm{K}^{+}, \mathrm{Ca}^{2+}$, $\mathrm{SB}, \mathrm{CTCe}$ e $\mathrm{m} \%$ se ajustaram ao modelo esférico e o $\mathrm{Mg}^{2+}$, $\mathrm{CTC}_{\mathrm{pH} 7}$ e V\% ao modelo exponencial (Tabela 4). Esses resultados concordam com os observados por várias pesquisas que indicam que o modelo esférico ocorre em maior frequencia em relação aos outros modelos (SOUZA; COGO; VIEIRA, 1997; SOUZA et. al., 2004; GREGO; VIEIRA, 2005; GOMES et al., 2008). Já Corá et al. (2004) e Corrêa; Tavares e Uribe-Opazo (2009), encontraram resultados contrários aos deste estudo, tendo em vista que na maioria das vezes o ajuste do modelo foi do tipo exponencial. O coeficiente de determinação $\left(\mathrm{R}^{2}\right)$

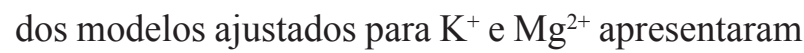
valores de 0,86 e 0,90 respectivamente, já as demais características da fertilidade do solo apresentaram $\mathrm{R}^{2}$ superiores que variaram entre 0,95 e 0,99 .

$\mathrm{Na}$ análise do grau de dependência espacial (GDE) utilizou-se a classificação recomendada por Robertson (2008), observou-se que apenas os atributos químicos M.O., pH, $\mathrm{P}$ e $\mathrm{K}^{+}$apresentam dependência espacial moderada e os demais $\left(\mathrm{Al}^{3+}\right.$, $\mathrm{Ca}^{2+}, \mathrm{Mg}^{2+}, \mathrm{SB}, \mathrm{CTC}_{\mathrm{pH}}, \mathrm{CTC}_{\mathrm{e}}, \mathrm{V} \%$ e $\mathrm{m} \%$ ) forte dependência. Cambardella et al. (1994) verificaram que a forte dependência espacial de atributos do solo é atribuída aos fatores intrínsecos (mineralogia e textura), ao passo que aos extrínsecos (manejo do solo) atribui-se fraca dependência. Assim, a forte dependência espacial encontrada para as características químicos do solo em estudo pode ser decorrente de qualquer um dos fatores de formação do solo, principalmente o material de origem e relevo, enquanto que a dependência 
espacial moderada seria devido à homogeneização do solo, confirmado esta hipótese quando se analisa a amplitude de variação dos atributos químicos M.O., $\mathrm{pH}, \mathrm{P}$ e $\mathrm{K}^{+}$que apresentam $\mathrm{CV}$ mais baixo (Tabela 2) em relação aos outros atributos e, o GDE foi moderado. Situação semelhante foi observada por Cavalcante et al. (2007) estudando diferentes sistemas de uso e manejo do solo.

$\mathrm{O}$ alcance (A) de um atributo garante que todos os pontos dentro de um círculo com este raio são tão similares que podem ser usados para estimar valores para qualquer ponto entre eles (MACHADO et al., 2007). Os atributos químicos do solo apresentaram diferentes alcances, sendo que os teores de M.O e de $\mathrm{Mg}^{2+}$ apresentaram os maiores valores de alcances, isso é 1.002 e $1.071 \mathrm{~m}$, respectivamente, enquanto que os teores de $\mathrm{P}$ e $\mathrm{CTC}_{\mathrm{pH}} 7$ tiveram os menores alcances, 449 e $331 \mathrm{~m}$, respectivamente. A menor continuidade espacial dos teores de $\mathrm{Pe} \mathrm{CTC}_{\mathrm{pH} 7}$ pode ser explicada pela aplicação de adubos e calcário de forma descontinua já que, as áreas de Floresta, Capoeira e Cerradão não receberam nenhum tipo de fertilização e correção. Os valores altos de alcance dos demais atributos, que variaram de 1.002 a 1.070 $\mathrm{m}$ indica que apesar dos diferentes manejos do solo realizado ao longo do tempo, tais como: aplicações de fertilizantes e calcário, preparo convencional do solo com arações e gradagens nas áreas de Solo nu, Cultivo agrícola e Pastagem, não foram capazes de interferir de modo a diminuir a continuidade na distribuição espacial dos atributos M.O., $\mathrm{Al}^{3+}, \mathrm{K}^{+}$, $\mathrm{Mg}^{2+}, \mathrm{SB}$ e CTC $\mathrm{e}_{\mathrm{e}}$. Os valores do alcance relativos aos semivariogramas têm uma importância considerável na determinação do limite da dependência espacial, podendo ser também um indicativo do intervalo entre unidades de mapeamento de solos (GREGO; VIEIRA, 2005).

A magnitude do efeito pepita $\left(\mathrm{C}_{0}\right)$ é importante na Krigagem, pois, quanto maior for a diferença do $\mathrm{C}_{0}$ em relação ao patamar $\left(\mathrm{C}_{0}+\mathrm{C}\right)$ do semivariograma, maior a continuidade do fenômeno, menor a variância da estimativa, ou maior a confiança que se pode ter na estimativa (ISAAKS; SRIVASTAVA, 1989). A partir dos modelos obtidos, para os semivariogramas ajustados para cada atributo químico do estudo, estimaram-se os valores das variáveis químicas em locais não amostrados por meio do método de interpolação geoestatístico de Krigagem. Deste modo, com os valores estimados foi possível construir mapas de isolinhas que expressam a variabilidade das variáveis avaliadas na área em estudo (Figuras 2 e 3).

Uma analise visual dos mapas gerados permitiu identificar zonas homogêneas em função da classe de uso e manejo de fertilização do solo. Essa divisão em zonas permite a utilização de manejos de adubação e correção específicos de modo a garantir um uso racional das áreas sob influencia de diferentes situações de uso. 

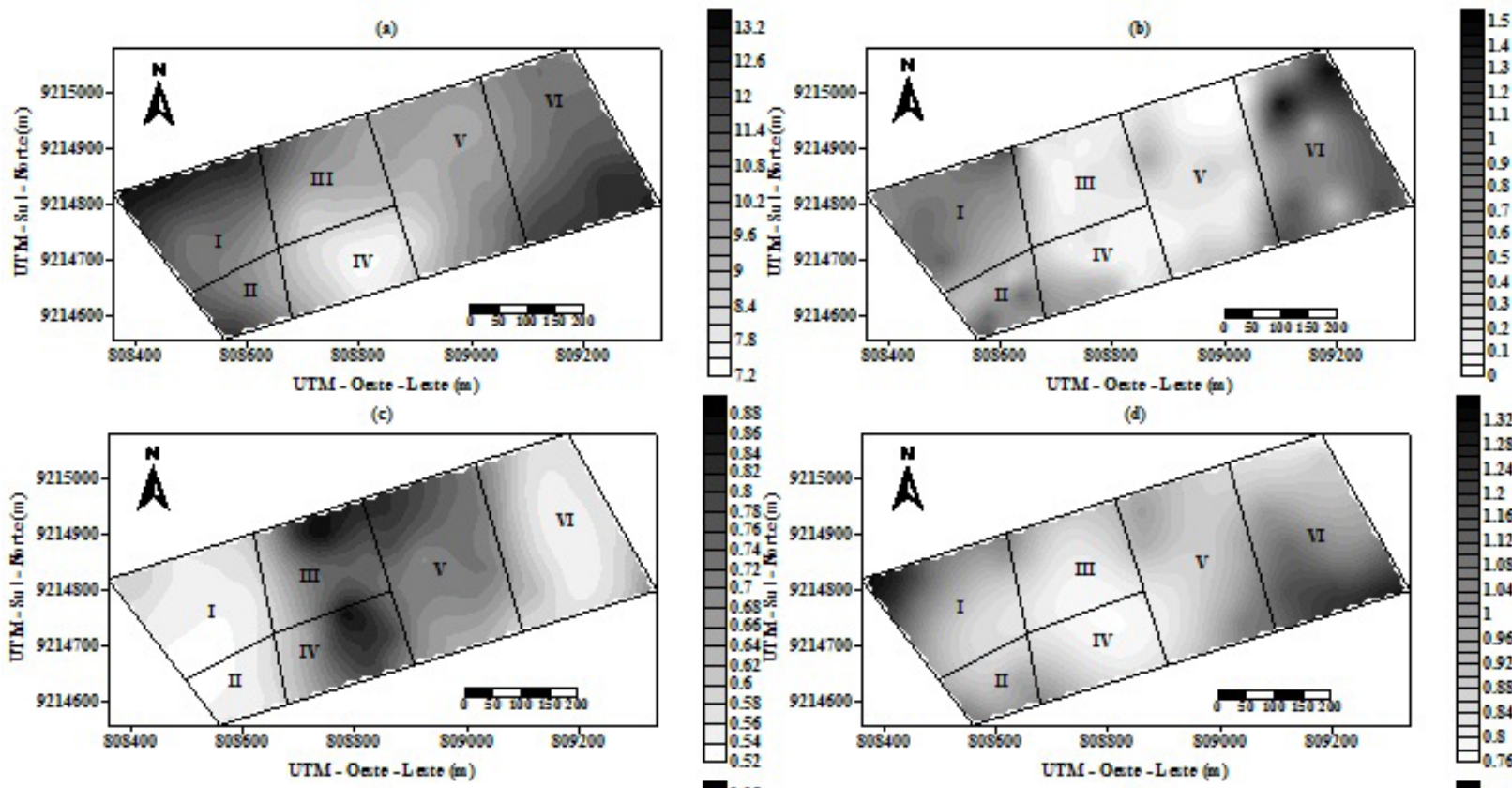

(d)
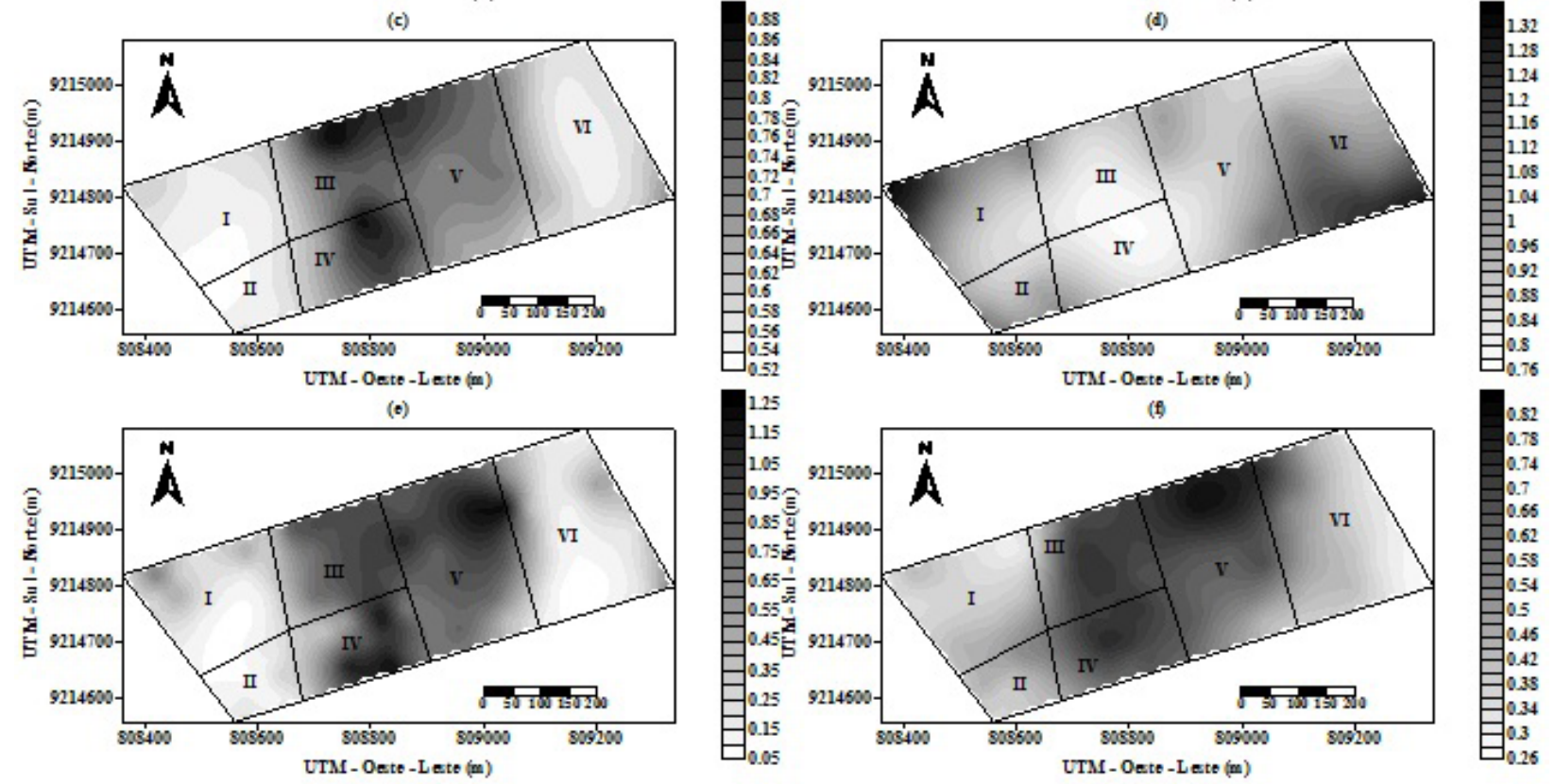

Figura 2. Mapas de isolinhas da distribuição espacial dos atributos químicos do solo, (a) M.O. ( $\left.\mathrm{g} \mathrm{dm}^{-3}\right),(\mathrm{b}) \mathrm{Al}^{3+}\left(\mathrm{cmol}_{\mathrm{c}}\right.$ $\left.\mathrm{dm}^{-3}\right)$, (c) $\mathrm{P}\left(\mathrm{mg} \mathrm{dm}^{-3}\right)$, (d) $\mathrm{K}^{+}\left(\mathrm{mg} \mathrm{dm}^{-3}\right)$, (e) $\mathrm{Ca}^{2+}\left(\mathrm{cmol} \mathrm{dm}^{-3}\right)$ e (f) $\mathrm{Mg}^{2+}\left(\mathrm{cmol}_{\mathrm{c}} \mathrm{dm}^{-3}\right)$ em função das classes de uso do solo, (I) Floresta, (II) Capoeira, (III) Solo nu, (IV) Cultivo agrícola, (V) Pastagem e (VI) Cerradão, em zona de transição Cerrado-Amazônia . 

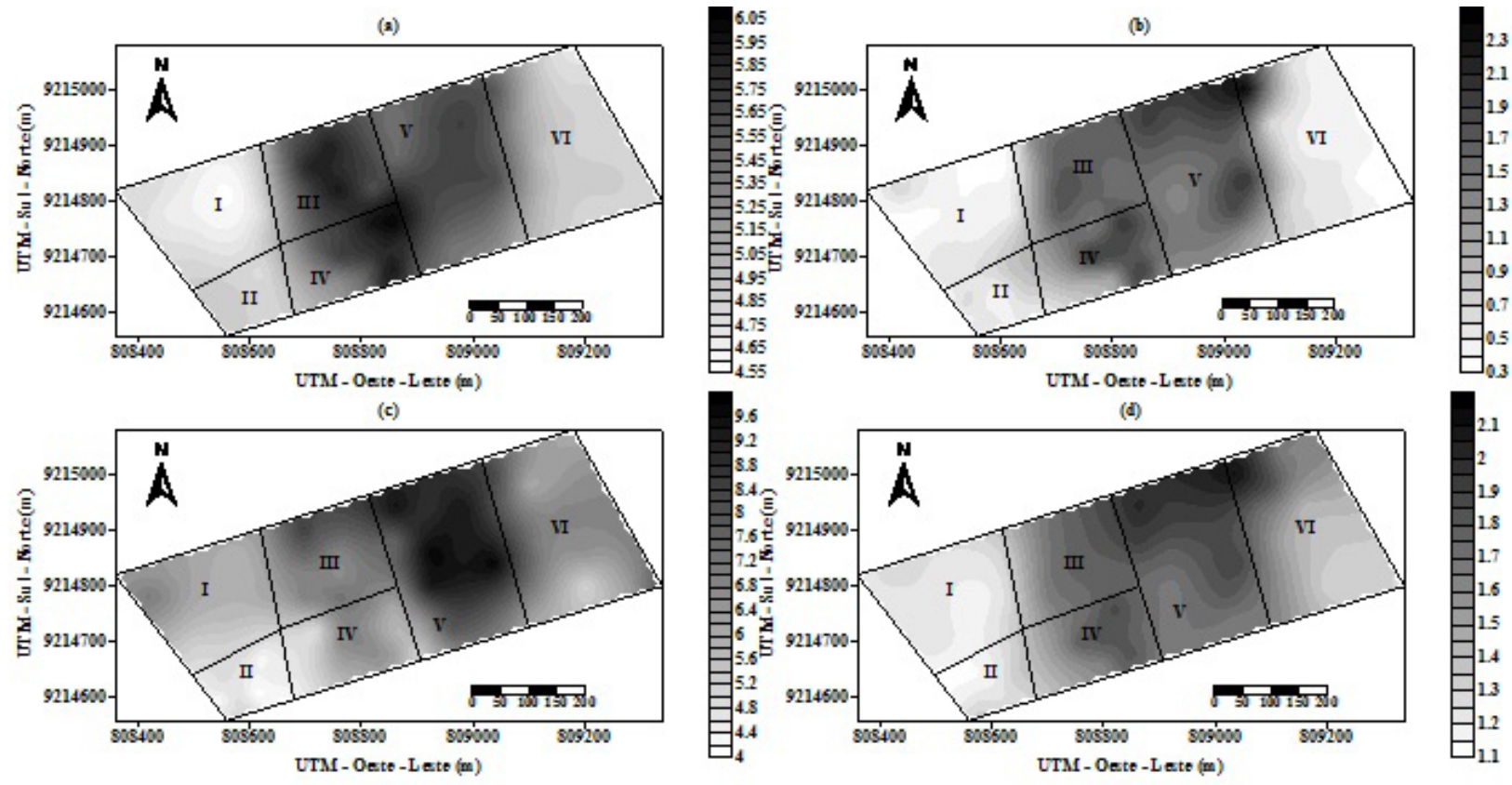

(o)
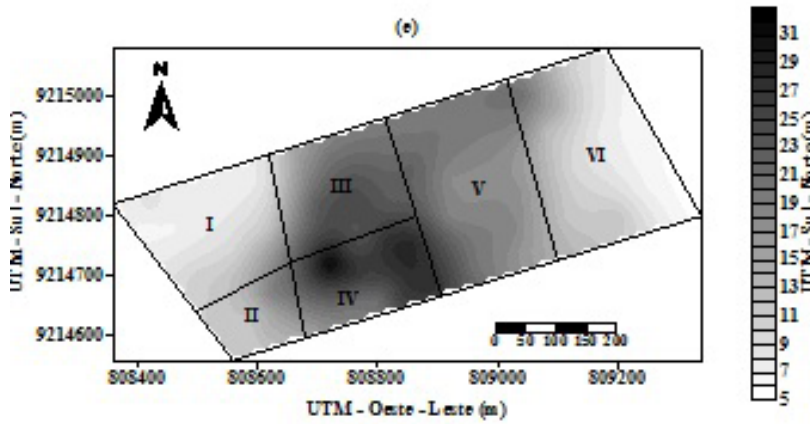

(f)

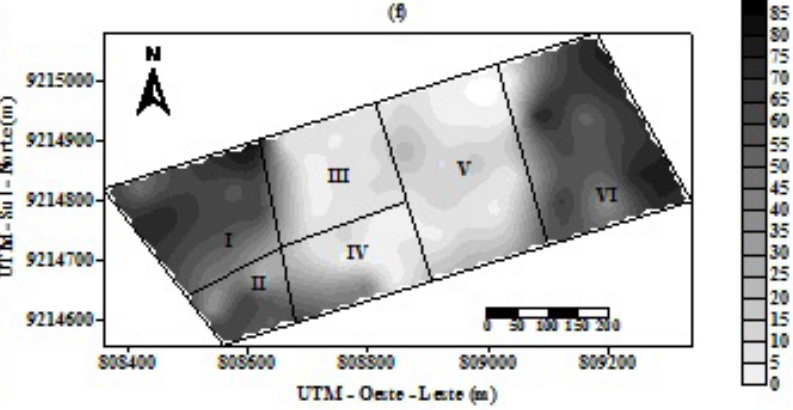

Figura 3. Mapas de isolinhas da distribuição espacial dos valores de, (a) pH $\left(\mathrm{CaCl}^{2}\right)$, (b) Soma de bases $\left(\mathrm{cmol}_{\mathrm{c}} \mathrm{dm}^{-3}\right)$, (c) $\mathrm{CTC}_{\mathrm{pH} 7}\left(\mathrm{cmol}_{\mathrm{c}} \mathrm{dm}^{-3}\right)$, (d) $\mathrm{CTC}_{\mathrm{e}}\left(\mathrm{cmol}_{\mathrm{c}} \mathrm{dm}^{-3}\right)$, (e) $\mathrm{V} \%$, (f) $\mathrm{m} \%$, em função das classes de uso do solo, (I) Floresta, (II) Capoeira, (III) Solo nu, (IV) Cultivo agrícola, (V) Pastagem e (VI) Cerradão, em zona de transição CerradoAmazônia .

\section{Conclusões}

O estudo dos distintos usos e manejos do solo, associado à análise da variabilidade espacial dos atributos químicos de Neossolo Quartzarênico Órtico típico na zona de transição do Bioma Cerrado e Amazônico permitiu a visualização e identificação, através dos mapas, de zonas homogêneas de manejo com limites bem definidos entre elas. $\mathrm{O}$ que admiti o uso de tecnologias que viabilizam a adoção de técnicas de agricultura de precisão e de conservação ambiental. As áreas ocupadas por Cultivo agrícola e Pastagem bem como a área de Solo nu, apresentaram distribuição espacial mais homogênea entre esses locais, que é o resultado das práticas de manejo localizadas, principalmente pela correção e adubação mineral. Já as áreas de Floresta, Capoeira e Cerradão se assemelharam quanto às características químicas do solo que pode ser resultado de fatores naturais (material de origem, textura, etc) e ambientais (cobertura vegetal).

\section{Agradecimentos}

Ao CNPq e Capes pelas bolsas concedidas aos autores e a Universidade Federal do Tocantins pelo apoio. 


\section{Referências}

CAMBARDELLA, C. A.; MOORMAN, T. B.; NOVAK, J. M.; PARKIN, T. B.; KARLEN, D. L.; TURCO, R. F.; KONOPKA, A. E. Field-scale variability of soil properties in Central Iowa Soils. Soil Science Society of America Journal, Madison, v. 58, n. 5, p. 1501-1511, 1994.

CARVALHO, J. R. P. de; SILVEIRA, P. M. da; VIEIRA, S. R. Geoestatística na determinação da variabilidade espacial de características químicas do solo sob diferentes preparos. Pesquisa Agropecuária Brasileira, Brasília, v. 37, n. 8, p. 1151-1159, 2002.

CAVAlCANTE, E. G. S.; ALVES, M. C.; SOUZA, Z. M. de; PEREIRA, G. T. Variabilidade espacial de atributos químicos do solo sob diferentes usos e manejos. Revista Brasileira de Ciência do Solo, Viçosa, v. 31, n. 6, p. 1329-1339, 2007.

COMISSÃO DE FERTILIDADE DO SOLO DO ESTADO DE MINAS GERAIS - CFSMG. Recomendações para o uso de corretivos e fertilizantes em Minas Gerais - 5a aproximação. Viçosa, 1999. 359 p.

CORÁ, J. E.; ARAUJO, A. V.; PEREIRA, G. T.; BERALDO, J. M. G. Variabilidade espacial de atributos do solo para adoção do sistema de agricultura de precisão na cultura de cana-de-açúcar. Revista Brasileira de Ciência do Solo, Viçosa, v. 28, n. 6, p. 1013-1021, 2004.

CORÁ, J. E.; BERALDO, J. M. G. Variabilidade espacial de atributos do solo antes e após calagem e fosfatagem em doses variadas na cultura de cana-de-açúcar. Engenharia Agrícola, Jaboticabal, v. 26, n. 2, p. 374-387, 2006.

CORRÊA, A. N.; TAVARES, M. H. F.; URIBE-OPAZO, M. A. Variabilidade espacial de atributos físicos do solo e seus efeitos sobre a produtividade do trigo. Semina: Ciências Agrárias, Londrina, v. 30, n. 1, p. 81-94, 2009.

EMPRESA BRASILEIRA DE PESQUISA AGROPECUÁRIA - EMBRAPA. Sistema brasileiro de classificação de solos. Brasília: Embrapa Produção de Informação, 2006. 306 p.

. Manual de análises de solo, planta e fertilizante. Brasília: Embrapa Produção de Informação, 1999. 412 p.

GOLDEN SOFTWARE. Surfer for Windows version 8.0. Colorado: Golden, 2002.

GOMES, F. P. A estatística moderna na pesquisa agropecuária. Piracicaba: POTAFOS, 1984. 160 p.

GOMES, J. B. V.; BOLFE, E. L.; CURI, N.; FONTES, H. R;. BARRETO, A. C.; VIANA, R. D. Variabilidade espacial de atributos de solos em unidades de manejo em área piloto de produção integrada de coco. Revista
Brasileira de Ciência do Solo, Viçosa, v. 32, n. 6, p. 2471-2482, 2008.

GOOVAERTS, P. Geostatistical tools for characterizing the spatial variability of microbiological and physicochemical soil properties. Biology and Fertility of Soils, Florence, v. 27, n. 4, p. 315-334, 1998.

GREGO, C. R.; VIEIRA, S. R. Variabilidade espacial de propriedades físicas do solo em uma parcela experimental. Revista Brasileira de Ciência do Solo, Viçosa, v. 29, n. 2 , p. 169-177, 2005.

ISAAKS, E. H.; SRIVASTAVA, R. M. An introduction to applied geoestatistics. New York: Oxford University, 1989. $561 \mathrm{p}$.

KÖPPEN, W. M. Climatologia: con un studio de los climas de la terra. México: Fondo de Cultura Económica, 1948. $478 \mathrm{p}$.

LI, H.; LASCANO, R. J.; BOOKER, J.; WILSON, L. T.; BRONSON, K. F.; SEGARRA, E. State-space description of heterogeneity: water and nitrogen use in cotton. Soil Science Society of America Journal, Madison, v. 66, n. 2, p. 585-595, 2002.

LITTLE, T. M.; HILLS, F. J. Agricultural experimentation. New York: John Wiley \& Sons, 1978. 350 p.

MACHADO, L. O.; LANA, A. M. Q.; LANA, R. M. Q.; GUIMARÃES, E. C.; FERREIRA, C. V. Variabilidade espacial de atributos químicos do solo em áreas sob sistema plantio convencional. Revista Brasileira de Ciência do Solo, Viçosa, v. 31, n. 3, p. 591-599, 2007.

MARCHIORI JUNIOR, M.; MELO, W. J. de. Alterações na matéria orgânica e na biomassa microbiana em solo de mata natural submetido a diferentes manejos. Pesquisa Agropecuária Brasileira, Brasília, v. 35, n. 6, p. 1177 1182, 2000.

MONTANARI, R.; PEREIRA, G. T.; JÚNIOR, J. M.; SOUZA, Z. M.; PAZETO, R. J.; CAMARGO, L.A. Variabilidade espacial de atributos químicos em Latossolo e Argissolos. Ciência Rural, Santa Maria, v. 38 , n. 5, 2008.

OLIVEIRA, J. J.; CHAVES, L. H. G.; QUEIROZ, J. E.; LUNA, J. G. Variabilidade espacial de propriedades químicas em um solo salino-sódico. Revista Brasileira de Ciência do Solo, Viçosa, v. 23, n. 4, p. 783-789, 1999.

ROBERTSON, G. P. GS+: geostatistics for the environmental sciences - GS+ User's guide. Plainwell: Gamma Design Soffware, 1998. 152 p.

SANTOS, A. C.; SALCEDO, I. H.; CANDEIAS, A. L. B. Relação entre o relevo e as classes texturais do solo na microbacia hidrográfica de Vaca Brava, PB. Revista Brasileira de Cartografia, Rio de Janeiro, v. 54, n. 1, p. 
86-94, 2002.

SCHAFFRATH, V. R.; TORMENA, C. A.; FIDALSKI, J.; GONCALVES, A. C. A. Variabilidade e correlação espacial de propriedades físicas de solo sob plantio direto e preparo convencional. Revista Brasileira de Ciência do Solo, Viçosa, v. 32, n. 4, p. 1369-1377, 2008.

SILVA, F. A. S. Software assistat: assistência estatística. Versão 7.5 beta. Campina Grande: UAEG-CTRN-UFCG, 2008.

SILVA, V. R.; REICHERT, J. M.; STORCK, L.; FEIJO, $\mathrm{S}$. Variabilidade espacial das características químicas do solo e produtividade de milho em um argissolo vermelhoamarelo distrófico arênico. Revista Brasileira de Ciência do Solo, Viçosa, v. 27, n. 6, p. 1013-1020, 2003.

SOUZA, L. S.; COGO, N. P.; VIEIRA, S. R. Variabilidade de propriedades físicas e químicas do solo em um pomar cítrico. Revista Brasileira de Ciência do Solo, Viçosa, v. 21, n. 3, p. 367-372, 1997.

SOUZA, Z. M.; MARQUES JUNIOR, J.; PEREIRA, G. T.; MOREIRA, L. F. Variabilidade espacial do $\mathrm{pH}, \mathrm{Ca}$, $\mathrm{Mg}$ e V\% do solo em diferentes formas do relevo sob cultivo de cana-de-açúcar. Ciência Rural, Santa Maria, v. 34, n. 6, p. 1763-1771, 2004.

SU, Y. Z.; ZHAO, H. L.; ZHANG, T. H.; ZHAO, X. Y. Soil properties following cultivation and non-grazing of a semi-arid sandy grassland in Northern China. Soil Tillage Research, Amsterdam, v. 75, n. 1, p. 27-36, 2004.

VIEIRA, S. R. Geoestatística em estudos de variabilidade espacial do solo. In: NOVAIS, R. F.; ALVAREZ, V. H.; SCHAEFER, C. E. G. R. (Ed.). Tópicos em ciência do solo. Viçosa: Sociedade Brasileira de Ciência do Solo, 2000. v. 1, p. 1-53. 